\title{
REVISIÓN DE EXPERTOS PARA MEDIR LA CALIDAD DE OBJETOS DE APRENDIZAJE
}

\author{
EXPERT REVIEW FOR MEASURING THE QUALITY OF LEARNING OBJECTS
}

\author{
Néstor Duque ${ }^{1}$, Alejandra Ospina², Valentina Tabares ${ }^{3}$, Demetrio Ovalle ${ }^{4}$ \\ Recibido para publicación: 19 de mayo de 2014 - Aceptado para publicación: 15 julio de 2014
}

\section{RESUMEN}

Los Objetos de Aprendizaje (OAs) han originado un nuevo modelo conceptual para organizar el contenido en contextos educativos basados en e-learning. Debido a su importante aporte a los procesos de enseñanza/ aprendizaje, se requiere de esfuerzos que permitan establecer estrategias para determinar y asegurar su calidad. La calidad en este contexto se entiende como el cumplimiento de un conjunto de características deseables del $\mathrm{OA}$, desde diferentes dimensiones, tales como educativa, de contenido, estética, funcional y de metadatos. Una propuesta para revisión de OAs por parte de expertos, es presentada en el presente trabajo, donde se define un conjunto de 8 criterios orientados a obtener una visión general del OA, y que permitirán tomar decisiones frente a estrategias para el aseguramiento de la calidad de los recursos.

Esta propuesta fue aplicada en la evaluación de OAs disponibles a través de la federación FROAC, evidenciando problemas de calidad de estos recursos, y con ello la necesidad de seguir trabajando en mejorar los procesos de construcción de estos recursos educativos.

Palabras Clave: Objetos de Aprendizaje, federaciones de repositorios, evaluación de calidad de OAs, expertos

\begin{abstract}
Learning Objects (LOs) have created a new conceptual model for organizing content in educational settings based on e-learning. Because of its important contribution to the teaching / learning processes, it is required to establish strategies to identify and ensure its quality.

Quality in this context is understood as the fulfillment of a set of desirable characteristics of OA from different dimensions, such as education, content, aesthetics, functional and metadata. A proposal for revision of AOs by experts is presented in this paper, where a set of 8 criteria for obtaining an overview of the OA is defined, and that will make decisions about strategies for quality assurance of resources.

This proposal was applied in the evaluation of LOs available through FROAC federation, showing quality problems these resources, and thus the need to continue working to improve the processes of construction of these educational resources.
\end{abstract}

Keywords: Learning Objects, federations of repositories of LOs quality evaluation, experts

1Universidad Nacional de Colombia - Sede Manizales. Colombia. ndduqueme@unal.edu.co.

Universidad Nacional de Colombia - Sede Manizales. Colombia. aospinah@unal.edu.co.

3Universidad Nacional de Colombia - Sede Manizales. Colombia. vtabaresm@unal.edu.co.

${ }^{4}$ Universidad Nacional de Colombia - Sede Medellín. dovalle@unal.edu.co. 


\section{INTRODUCCIÓN}

EL uso de recursos digitales para el apoyo de los procesos de enseñanza-aprendizaje, bajo la figura de Objetos de Aprendizaje (OA), que son unidades educativas acompañadas de sus metadatos, ha tomado un papel fundamental potencializando estos procesos [1].

Debido a la gran cantidad de OAs que se encuentran disponibles, los usuarios cada vez buscan mejores recursos que apoyen su proceso de formación. Sin embargo, se han hecho evidentes diferentes problemas asociados a la calidad de los recursos, convirtiéndose en un tema que requiere abordarse desde diferentes enfoques [2] [3].

La razón principal para preocuparse por la calidad de los OAs, es que se trata de recursos de apoyo al aprendizaje. Aun cuando no es el único factor que afecta a los procesos de enseñanza y aprendizaje, la carencia de controles de calidad en estos recursos puede afectar al proceso y sus resultados en los aprendices, además que la existencia de recursos de baja calidad y la carencia de evaluaciones sistemáticas, podría provocar el desinterés o la desconfianza en su uso [4] [5].

Miles de OAs son almacenados en bibliotecas digitales denominadas repositorios de OAs (ROAs) que deben seguir estándares con el fin de garantizar el acceso por parte de estudiantes y profesores alrededor del mundo, además se cuenta con Federaciones de Repositorios que permiten un punto único de acceso a los recursos almacenados en diferentes repositorios. Sin embargo, no es suficiente con que estos materiales educativos estén disponibles, se requiere que cumplan con un alto nivel de calidad, de modo que permita mayores posibilidades de lograr los objetivos de aprendizaje esperados en los estudiantes [6].

Específicamente, en el marco de la propuesta de la Federación de Repositorios de Objetos de Aprendizaje Colombia (FROAC), que es una iniciativa que ofrecer aplicaciones y servicios producto de procesos de investigación, lo que además la convierte en una plataforma experimental de gran valor para la comunidad involucrada en su desarrollo [7], se ha enfrentado el tema de la evaluación de OAs desde diferentes enfoques, como el presentado en [8] que propone métricas para la evaluación automática de metadatos, el presentado en [9] que plantea una estrategia para valoración de OAs teniendo en cuenta la percepción de los usuarios, y el propuesto en este artículo, donde se aprovecha la revisión de expertos, que aplican un instrumento que abarca criterios que permiten determinar el nivel de calidad de los OAs analizados.

La propuesta presentada es aplicada con los OAs disponibles a través de FROAC, determinando el nivel de cumplimiento que tienen estos recursos de los criterios de calidad evaluados. Los resultados demuestran la necesidad de seguir trabajando en este tema y de mejorar tanto los procesos de creación y actualización de los OAs, como los relacionados con la evaluación y aseguramiento de la calidad.

En la sección dos del artículo se presenta una revisión general de algunas propuestas relacionadas con la evaluación de OAs, en la sección tres se expone la propuesta de evaluación de objetos de aprendizaje desde un enfoque de revisión de experto, en la sección cuatro se muestran la validación y resultados obtenidos a través de la aplicación en FROAC, y finalmente en la sección cinco se presentan conclusiones y el trabajo futuro.

\section{TRABAJOS RELACIONADOS}

El uso de los OAs ha permitido transformar los contenidos digitales en unidades independientes con un enfoque altamente educativo, es por ello que su calidad se ha vuelto un tema de gran importancia. 
La calidad de un OA es entendida como un conjunto de criterios que permiten establecer su nivel de pertinencia en los procesos de enseñanza-aprendizaje, es decir, el grado de utilidad respecto al logro de las metas pedagógicas planteadas en el objetivo del OA [10]. Se plantea que la evaluación de la calidad de los recursos educativos puede hacerse desde dos aspectos: El producto (objeto de aprendizaje) y el proceso (desarrollo del objeto) [4].

La evaluación desde un enfoque basado en aspectos cualitativos a los cuales se les añade una ponderación cuantitativa que permite su aplicación en diferentes áreas del conocimiento es presentada en [11], mientras que algunos autores centran su investigación en mostrar que la calidad de los OAs se basa en elementos tecnológicos (reutilización y adaptabilidad), pedagógicos (posibilidad de experimentación y posibilidad de adaptación) y los elementos de contenido (nivel de detalle y complejidad del tema) [12].

Por otro lado, en [13] se sugieren una metodología para evaluar la calidad de los OAs teniendo en cuenta cuestiones pedagógicas y de usabilidad en cuanto al diseño de contenidos y de la navegación. Estos criterios son formalizados en una herramienta que guiará a expertos durante la revisión de los OAs y la asignación a cada elemento de un valor numérico dentro de un rango predefinido.

Finalmente se presenta el LORI (Learning Object Review Instrument), un instrumento para la valoración de OAs por parte de expertos, de forma individual o colaborativa, que permite evaluar las siguientes dimensiones: Calidad del contenido, alineamiento con los objetivos de aprendizaje, retroalimentación y adaptación, motivación, presentación, usabilidad de la interacción, accesibilidad, reusabilidad, cumplimiento de estándares. Cada criterio es evaluado en una escala de cinco niveles. Este instrumento es ampliamente conocido y aplicado en repositorios como MERLOT y CLOE [14] [15].
Esta revisión evidencia el creciente interés en la comunidad en cuanto a temas relacionados con la evaluación de la calidad de OAs, específicamente desde el enfoque de revisión de expertos. Se presentan trabajos en los cuales, se proponen instrumentos para la revisión de OAs, pero en algunos casos no evalúan los recursos completamente, son demasiado largos o no permiten calcular un indicador global de la calidad del OA. Lo anterior implica, que aun se requiere de esfuerzos que permitan mejorar y aplicar con mayor efectividad el proceso de evaluación de recursos educativos digitales.

\section{REVISIÓN DE EXPERTOS PARA EVALUAR OAS}

Aprovechando la experiencia obtenida en procesos de evaluación previos de los OAs disponibles a través de FROAC [16], desde enfoques como el de evaluación automática de metadatos presentado en [8] y el de análisis de la percepción de usuarios expuesto en [9], se proponen un conjunto de criterios, con el fin de determinar el nivel de calidad del OA a partir de la revisión de expertos.

Algunos repositorios realizan la evaluación de sus OAs únicamente antes de incorporarlos y permitir su visualización, pero son muchos los recursos que ya se encuentran disponibles y no cumplen con los criterios de calidad. Por lo tanto, esta propuesta está orientada a la evaluación tanto de recursos que ya se encuentran almacenados en los repositorios, como a aquellos que están en un proceso de revisión inicial. Específicamente para esta propuesta, se le solicita a un experto, que es considerado una persona cuya experiencia, capacidad y competencia, le permiten realizar un análisis frente a una serie de criterios planteados y emitir una valoración [10], que a través de un instrumento revise los OAs asignados. Este instrumento, se enfoca en cinco dimensiones para analizar el OA que son: Educativa, de Contenido, Estética, Funcional y de Metadatos; las cuales se dividen en 8 criterios, que se muestran con 
detalle en la Tabla 1, y que permiten tener una visión clara y precisa de la calidad del objeto.

A partir de los criterios establecidos, se definió un conjunto de 15 preguntas, que serán diligenciadas por el experto, a través de un formulario como el presentado en la Figura 1.

El experto debe responder a las preguntas con un valor numérico en una escala entre 1 y 5 , donde 1 representa el peor valor y 5 el mejor. Después de obtener las respuestas del experto, éstas se ponderan con las dadas por otros expertos para el mismo OA y se obtiene un valor para cada criterio evaluado.

Dado que uno de los objetivos de esta propuesta, es su integración con evaluaciones realizadas desde otros enfoques, se realiza una conversión

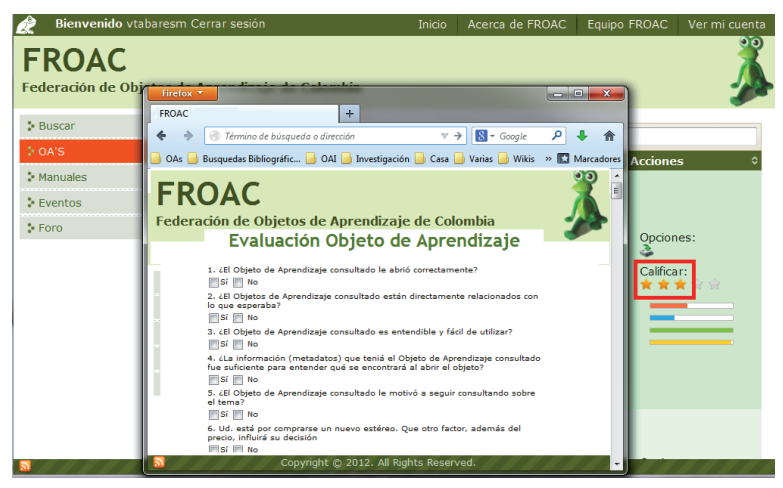

Figura 1. Evaluación Expertos
Tabla 1. Dimensiones y criterios de evaluación

\section{Dimensión Educativa}

Efectividad Potencial: Asociado al nivel de eficiencia que puede llegar a tener el $\mathrm{OA}$ en el proceso de enseñanza-aprendizaje, analizando si se pueden evidenciar los objetivos educativos y si la estructura del OA apoya el aprendizaje del tema.

\section{Dimensión de Contenido}

Pertinencia y Rigurosidad: Evalúa el OA desde la parte semántica y sintáctica, buscando que se presente de una manera pertinente para que no se afecte el aprendizaje.

\section{Dimensión de Estética}

Diseño Visual: Asociado al contenido visual que se encuentra en el OA como imágenes, letra, tamaño, color, entre otros; ya que este influencia el proceso de aprendizaje.

\section{Dimensión de Funcional}

Reusabilidad: Hace referencia a la capacidad del OA de ser usado en diferentes contextos, cumpliendo una de sus principales características.

Facilidad de Uso: Analiza el nivel de complejidad que tiene el objeto a la hora de interactuar con él.

Facilidad de Acceso: Se analiza si existe facilidad para abrir y usar el OA o si se requiere de software especializado para su visualización.

\section{Dimensión de Metadatos}

Completitud: Evalúa la capacidad que tienen los metadatos de describir el OA tanto como sea posible. Precisión: Busca determinar si los metadatos asociados al objeto si hacen referencia al contenido asociado.

a una escala entre 0 y 1 . También se ponderan los valores, obteniendo una medida que indica el nivel de calidad respecto las dimensiones evaluadas.
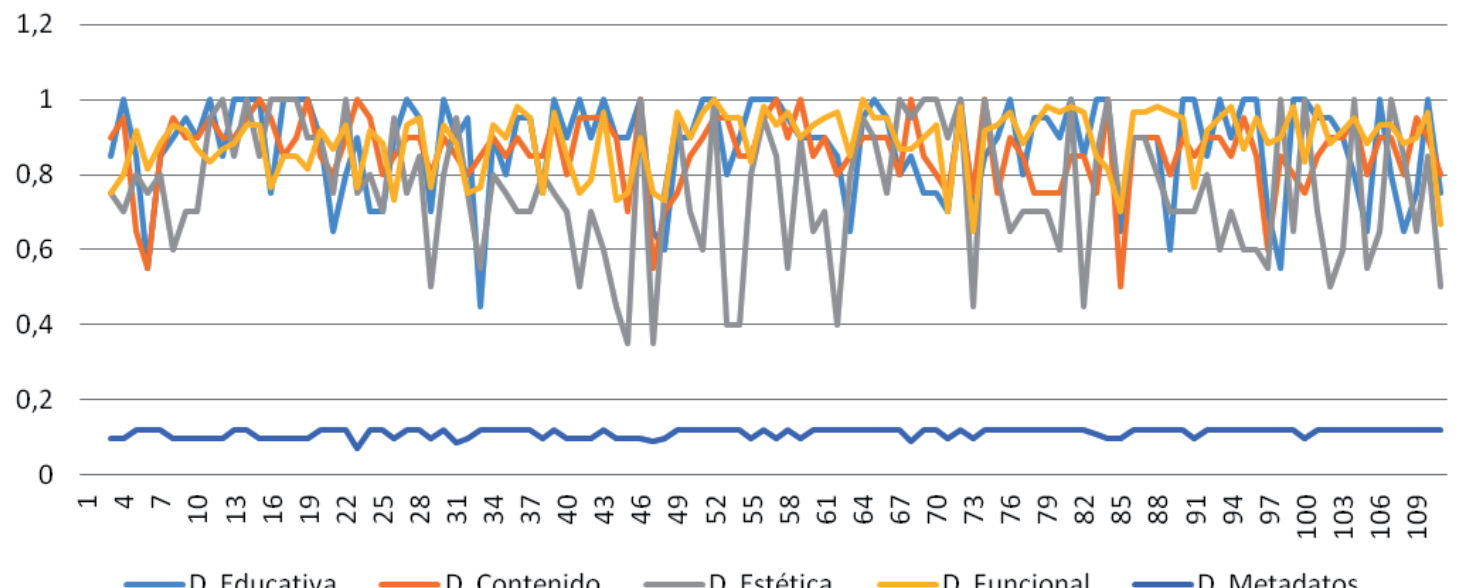

Figura 2. Resultados de Evaluación por Dimensión 


\section{EXPERIMENTACIÓNY RESULTADOS}

La estrategia de evaluación presentada, fue usada para evaluar un total de 109 OAs almacenados en FROAC, lo que permitió determinar su aplicabilidad, además validar con los expertos el instrumento diseñado.

Se consideró un grupo de expertos de 15 personas, entre los cuales estaban profesores y estudiantes de postgrado de la Universidad Nacional de Colombia - Sede Manizales, quienes durante un periodo de tiempo se encargaban de aplicar el formulario de evaluación al menos a 5 OAs que se les asignaron.

Adicional a los valores numéricos obtenidos, fue posible identificar a través de la revisión de expertos, problemas asociados a la disponibilidad de los recursos, lo que permitió mejorar el acceso a los OAs por parte de los usuarios.

En la Figura 2 se muestran los resultados para todos los OAs evaluados de acuerdo a las dimensiones propuestas y en la Figura 3 se pueden observar el porcentaje de cumplimiento de cada dimensión de acuerdo a todos los recursos evaluados.

En estas figuras se evidencia problemas en la dimensión de metadatos, mostrándola como la más baja de todas las dimensiones evaluadas. Esto puede indicar un problema en el proceso de etiquetado de los OAs, ya que estos se están

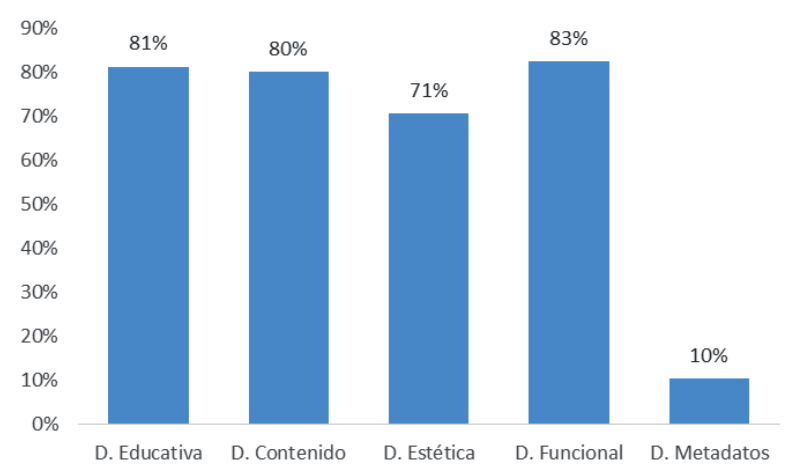

Figura 3. Porcentaje de Cumplimiento por Dimensión dejando incompletos o no están correspondiendo directamente a una descripción precisa del contenido.

También se hace notoria la buena calificación que tiene la dimensión educativa, mostrando con ello que se está cumpliendo con uno de los criterios de mayor importancia en cuanto a los OAs, dada su clara orientación hacia el apoyo de procesos de enseñanza-aprendizaje. Sin embargo, se identifican alrededor de 11 OAs que tienen una baja calificación en esta dimensión, lo que implica una revisión manual más detallada, que permita determinar si esos recursos deben ser actualizados y seguir haciendo parte de la federación, o descartarlos por problemas de baja calidad.

Finalmente, para obtener un valor numérico que permita estimar en términos generales el nivel de calidad de cada OA, se realizó ponderación de los resultados agrupados en dimensiones para cada recurso. Esta ponderación se hizo dando mayor peso a las dimensiones Educativa, de Contenido y Funcional. En la Tabla 2 se pueden observar las ponderaciones definidas para cada dimensión, de acuerdo al enfoque que se considera deben tener los OAs.

Tabla 2. Ponderación de las Dimensiones

\begin{tabular}{lc}
\hline \multicolumn{1}{c}{ Dimensión } & Ponderación \\
\hline Educativa & 0,3 \\
Contenido & 0,2 \\
Estética & 0,15 \\
Funcional & 0,2 \\
Metadatos & 0,15 \\
\hline
\end{tabular}

\section{CONCLUSIONES}

Es indispensable la evaluación de OAs que se encuentran disponibles a través de repositorios y federaciones, ya que esto permite identificar su nivel de calidad y tomar medidas orientadas a mejorar la experiencia de los usuarios, al ofrecerle solo recursos que aporten en su proceso de enseñanza-aprendizaje. 
Dado que la propuesta presentada en este artículo, se complementa con otros enfoques de evaluación de OA implementados en FROAC, es posible integrar los resultados para conseguir una evaluación general de los recursos desde diferentes perspectivas, representando un gran potencial en el proceso de aseguramiento de la calidad de los OAs.

Los resultados obtenidos, permitieron identificar que aun hay muchos problemas por resolver asociados a la calidad de los OAs, tanto desde aspectos educativos como aspectos técnicos asociados a los metadatos y a su funcionalidad.

Como trabajo futuro se espera aplicar la evaluación a un mayor número de OAs y con un grupo más amplio de expertos, además se está trabajando en la definición de perfiles de expertos que permita dar mayor peso a las preguntas relacionadas directamente con sus áreas de interés y desempeño, determinando un nivel de confianza para cada evaluador.

También se espera con las pruebas realizadas, poder identificar aspectos como el nivel de subjetividad de los evaluadores encargados e identificar la mejor forma de normalizar los resultados y poder obtener una calificación estándar que logre una mayor confianza en la calidad de los OAs.

\section{REFERENCIAS}

[1] D. A. Wiley, "Connecting learning objects to instructional design theory: A definition, a metaphor, and a taxonomy," vol. 2830, no. 435, pp. 1-35, 2001.

[2] X. Ochoa, "Learnometrics: Metrics for Learning Objects - Tesis Doctoral," Katholieke Universiteit Leuven, 2008.

[3] S. M. Massa, "Objetos de Aprendizaje: Metodología de Desarrollo y Evaluación de la Calidad," Universidad Nacional de La Plata, 2012.
[4] C. Vidal, A. Segura, and M. Prieto, "Calidad en Objetos de Aprendizaje," $V$ Simp. Pluridiscip. sobre Diseño y Evaluación Contenidos Educ. Reutil. SPEDECE 08, vol. 9126, no. parte 1, 2008.

[5] V. Tabares, N. Duque, and J. Moreno, "Análisis experimental de la utilidad en la recuperación de objetos de aprendizaje desde repositorios remotos," in Congreso Internacional en Ambientes Virtuales de Aprendizajes Accesibles y Adaptativos CAVA 2011, 2011.

[6] R. McGreal, "A Typology of Learning Object Repositories," in Handbook on Information Technologies for Education and Training, 2008, pp. $5-28$.

[7] V. Tabares, P. A. Rodríguez, N. D. Duque, and J. Moreno, "Modelo Integral de Federación de Objetos de Aprendizaje en Colombia - más que búsquedas centralizadas," in Séptima Conferencia Latinoamericana de Objetos y Tecnologías de Aprendizaje - LACLO 2012, 2012, vol. 3, no. 1, pp. 410-418.

[8] V. Tabares, P. Rodríguez, N. Duque, R. Vicari, and J. Moreno, "Multi-agent Model for Evaluation of Learning Objects from Repository Federations - ELO-index," Rev. Respuestas, no. 1, pp. 48-54, 2012.

[9] V. Tabares, N. Duque, and S. Baldiris, "Calidad de Objetos de Aprendizaje en FROAC desde la Percepción del Usuario," Congr. Int. en Ambient. Virtuales Aprendizajes Accesibles y Adapt. - CAVA 2013, vol. En Evaluac, 2013.

[10] V. Tabares, "Modelo por capas para Evaluación de la Calidad de Objetos de Aprendizaje en Repositorios de Objetos de Aprendizaje - Tesis de Maestría," Universidad Nacional de Colombia, 2013. 
[11] R. L. Gonzales, J. M. Arteaga, and F. A. Rodriguez, "La Calidad en los Objetos de Aprendizaje," pp. 352-357, 2006.

[12] C. Toll, "La calidad de los objetos de aprendizaje producidos en la universidad de las ciencias informáticas," pp. 1-18.

[13] E. Morales, A. Gil, and F. García, "Arquitectura para la Recuperación de Objetos de Aprendizaje de calidad en Repositorios Distribuidos," SCHA Sist. Hipermedia Colab. y Adapt. II Congr. Español Informática CEDI 2007, vol. 1, pp. 31-38, 2007.
[14] J. Nesbit, K. Belfer, and T. Leacock, "Learning Object Review Instrument (LORI) - User Manual," 2003.

[15] J. Vargo, J. C. Nesbit, K. Belfer, and A. Archambault, "Learning Object Evaluation: Computer-Mediated Collaboration and Inter-Rater Reliavility," Int. J. Comput. Appl., vol. 25, no. 3, 2003.

[16] V. Tabares, P. A. Rodríguez, and N. D. D. M, "Modelo Integral de Federación de Objetos de Aprendizaje." 